\title{
Escalating chronic kidney diseases of multi-factorial origin in Sri Lanka: causes, solutions, and recommendations
}

\author{
Sunil J. Wimalawansa
}

Received: 31 March 2014 / Accepted: 4 June 2014/Published online: 20 September 2014

(C) The Japanese Society for Hygiene 2014

\begin{abstract}
During the last two decades, Sri Lanka, located close to the equator, has experienced an escalating incidence of chronic kidney disease (CKD) of unknown aetiology (CKDue) in dry zonal areas. Similar incidences of unusual CKDs have been reported in the dry zonal, agricultural areas of several other equatorial countries. In Sri Lanka, the incidence of CKDue is highest in the North Central Province (NCP), where approximately $45 \%$ of the country's paddy fields are located. However, in recent years, the disease has spread into areas adjacent to as well as distant from the NCP. The cause of CKD in Sri Lanka is unknown, and may likely due to interactions of different potential agents; thus, CKD is of multi-factorial origin (CKD-mfo). These factors include, the negative effects from overuse of agrochemicals. Nevertheless, the potential interactions and synergism between probable agents have not been studied. This systematic review discusses the proposed hypotheses and causes of CKD-mfo in Sri Lanka, and ways to decrease the incidence of this disease and to eradicate it, and provide some recommendations. During the past decade, a number of groups have investigated this disorder using different methodologies and reported various correlations, but failed to find a cause. Research has focussed on the contamination of water with heavy metals, agrochemicals, hard water, algae, ionicity, climate change, and so forth. Nevertheless, the levels of any of the pollutants or conditions reported in water in NPC are inconsistent not correlated with the prevalence of the disease, and
\end{abstract}

S. J. Wimalawansa $(\bowtie)$

Cardio Metabolic Institute, 51 Veronica Avenue,

Somerset, NJ 08873, USA

e-mail: suniljw@hotmail.com are too low to be the sole cause of CKD-mfo. Meanwhile, several nephrotoxins prevalent in the region, including medications, leptospirosis, toxic herbs, illicit alcohol, locally grown tobacco, and petrochemicals, as well as the effects of changed habits occured over the past four decades have not been studied to date. Taken together, the geographical distribution and overall findings indicate that combinations of factors and/or their interactions are likely to precipitate CKD-mfo, which kills more than 5,000 people annually in Sri Lanka; most victims are middleaged male farmers. Much anecdotal evidence from this region suggests that consumption of contaminated water is the most likely source of this deadly disease. Although the aetiology is unknown, prevention of this "environmentally acquired" disease seems relatively straightforward. Solutions include (a) preventing environmental pollution, (b) stopping the irresponsible use and decreasing the usage of agrochemicals, and encouraging the use of environmentally friendly agricultural methods, (c) taking proper precautions when using agrochemicals and safe disposal of their containers, (d) changing the risky behaviour of farmers and educating them to preserve the environment, and (e) providing clean potable water to all affected regions. Implementing a well-coordinated, in-depth, region-wide, broad-based research study together with a long-term effective surveillance programme across the country is essential to curbing this disease. Unless firm actions are taken promptly, more than three million healthy people in the country, live in agricultural regions, are at risk for contracting CKD-mfo and succumb to premature deaths, which are preventable.

Keywords Agriculture - Agrochemicals - Behaviour · CKD · Contamination · Environment · Farming · Government · Micro-albumin · Pollution · Renal failure 


\section{Introduction}

During the past two decades, an epidemic of chronic kidney disease (CKD) has been escalating in the North Central Province (NCP) of Sri Lanka, which is located south of India. Approximately 100,000 people are currently affected, with 5,000 deaths occurring each year, attributed to an unusual form of CKD of unknown aetiology (CKDue), with an approximate $5 \%$ death rate per year amongst the affected individuals [1]. Most of these deaths occur in villages in the NCP and other drier regions, but the disease is spreading to other areas distant from this region [2]. In certain villages, more than $10 \%$ of the adult population has this terminal illness [3].

It is important to conduct a root cause analysis in such epidemics, but attempts over the past decades have failed $[1,3]$. Therefore, under these unusual circumstances, with on average 13 people dying prematurely of CKD-mfo each day and the failure to find a cause, taking the most cost-effective preventative action plan is the best way to protect people contracting this disease. However, if the policy makers opt to wait until a firm cause is discovered or to wait until everyone agreed on what to do, people will continue to die. Because finding the cause may take several more years, such delays would lead to thousands more preventable deaths. The needed actions include enhancing region-wide awareness and an education campaign to prevent environmental pollution, educating farmers to use agrochemicals responsibly and decrease their excessive usage, providing safe potable water. In parallel, it is important to engage in a ground-based, indepth comprehensive study and a nationwide surveillance programme to identify trends and root causes of this deadly disease.

This review provides an insight into current hypotheses and possible causes of CKD-mfo in Sri Lanka, based on the scientific evidence available to date, and ways to decrease the incidence and eventually overcome the disease. In this regard, a set of recommendations urges authorities to take immediate preventative measures to keep the condition under control, and aid in the prevention and elimination of the disease. As the basic conditions and practices remain similar, these recommendations also applicable to other countries that are affected by this disease. Data available to date support that this unusual form of renal failure is caused by a combination of factors working in synergy, with consumption of contaminated water being one of the most important factors. Although this disease has been identified previously with various names, including CKDu (unknown), CKDuo (unknown origin), and CKDue (unknown aetiology), etc., considering its multi-factorial aetiologies, it is most logical to name it as CKD of multifactorial origin (CKD-mfo).

\section{Uniqueness of the CKD-mfo}

Most of the common forms of CKD are caused by longstanding hypertension, diabetes, and in certain cases, glomerulonephritis [4-6]. The common form of CKD is distributed uniformly in cities and villages across Sri Lanka, which is similar to the distribution of CKD globally. On the other hand, diseases similar to CKD-mfo have been reported in several farming communities in agricultural societies, in developing countries and emerging economies in peri-equatorial regions. Worldwide, these CKDue epidemics are localised in the dry zones of agricultural societies, where the landscape is flat and the ground conditions are harsh, with little rain and prolonged dry periods and drought. Examples of somewhat similar CKDu conditions reported in other countries include Uddanam, in the agricultural belt of Andhra Pradesh [7]; unusual nephropathy, in China [8] and Nicaragua [9]; and Balkan endemic nephropathy, which affects rural villages in Serbia, Bulgaria, Romania, Croatia, and Bosnia [10, 11].

Worldwide, CKDs of unknown aetiologies are affecting the poorest communities in agricultural regions in dry zonal areas [12-14]. In many of these regions, CKDue has been reported for more than four decades without identification of any specific causative factor(s). Although ground conditions and the histopathologic features of these CKDs are somewhat similar, the postulated causes are different amongst these countries [8, 10, 15, 16]. While some have been attributed to natural (e.g. arsenic, fluoride) or man-made groundwater contamination (drilling, construction, etc.), others point towards haphazard use and overuse of agrochemicals. The types of agrochemicals used in these regions are generally similar, but the frequency and the amounts used vary markedly. Similar to Balkan endemic nephropathy and other chronic nephropathies of unknown origin occurring across the world, the underlining specific cause of CKD-mfo in Sri Lanka is not only unknown but may also take years to identify.

The CKD caused by hypertension and diabetes mostly affects those who are over 60 years of age. The prevalence of CKD due to these two common causes in the NCP is not different from the rest of the country (Wimalawansa, unpublished). Moreover, there is no evidence to suggest that the CKD-mfo is related to any of the commonly identifiable causes, has an immunogenic origin, or has a genetic background [3, 5, 17]. Nevertheless, in patients with CKD-mfo, especially those who are older than 50 years, hypertension and diabetes are likely to co-exist and may further aggravates the renal failure [5]. Therefore, the diagnostic criteria established by the Department of Health and its recommendations to exclude all patients with hypertension or diabetes [3] when CKD-mfo is diagnosed are inappropriate. 
In view of what is mentioned above, sub-optimal diagnostic criteria and approaches would lead to further underestimation of the prevalence of CKD-mfo, which deny affected patients to access to care and the right treatment [1]. Irrespective of the aetiology, due to the inherent pathological mechanisms, all CKD types lead to development of hypertension, and thus, these diseases commonly co-exist. Not surprisingly, recent studies suggest a higher incidence of hypertension amongst those with CKD-mfo [4, 18]. However, it is unknown whether the hypertension was pre-existing, caused by the renal failure, or contributed to the precipitation of CKD-mfo.

\section{Postulated causes for CKD-mfo}

Worldwide, CKD of unknown/uncertain aetiology has been reported after chronic exposure to certain heavy metals, including arsenic, cadmium, mercury, chromium, uranium, gold, platinum, silver, thallium, and antimony, as well as fluoride [4, 19, 20] and agrochemicals [7-11]. Despite the epidemiological studies carried out over the past 40 years, other than the reported associations and correlations (except arsenic in ground-water, Bihar, India), no causative factor has been identified for CKDu across the continent. Similarly, in Sri Lanka, data from food and water samples testing obtained from the affected regions are inconsistent and have failed to confirm higher levels or a identifiable pattern of any of these putative agents in relation to the geographical distribution of the disease in the country [3].

Similarly, blood and urine testing, and kidney biopsy samples from patients with this disease compared with control samples obtained within the region failed to provide conclusive evidence of a causative factor for CKDmfo [21]. The reported findings are weak, and interpretations are not irrefutable [21], which further supports the theory that the aetiology of this illness is multi-factorial [4, 22], and synergistic interactions between these chemicals and/or conditions leading to enhanced renal toxicity are possible. Nevertheless, overall data suggest that agrochemicals are one of the leading candidates in precipitating this disease. However, the specific components in agrochemicals, the exposure dose and duration, and how these components cause the disease (i.e. the mechanism of action) if any, are yet to be identified.

Renal failure can develop secondary to a variety of toxins, including snakebites [23], herbal toxins and chemicals [23], and immunological disorders, such as glomerulonephritis [6, 17, 23]. Irrespective of the causative factor(s) of CKD-mfo, the renal tubular damage occurs in patients at an early stage of the disease [17]; with time; it causes irreversible renal fibrosis leading to renal failure and premature death $[6,17,24]$. Once kidneys are fibrosed, the damage is irreversible, so prevention is the only way forward. In addition, patients with all forms of CKDs are more prone to experience other medical ailments, including depression, hypocalcaemia, renal bone disease, cancer, and lung and cardiovascular diseases [25, 26], and may die of any of these co-existing disorders.

Research indicates that the renal tubular damage occurring in the early stages of CKD is similar to kidney tubular damage caused by many other known nephrotoxins [17]. Whether there is a particular susceptibility or a genetic trait in those who develop this disease is unknown [17], but this appears unlikely. There are other pitfalls in some of the assumptions made in published reports. The urinary excretion of various toxins varies depending on the time of collection. Moreover, with decreasing renal functions, the excretion of a causative toxin(s) may in fact, decrease. Therefore, the validity of correlations of urinary levels of potential agents with the disease status is questionable. Similarly, the results of water quality testing vary greatly during the season and the timing of collection (i.e. during the rainy or dry season, before or after a rainfall, etc.). Thus, neither is a reliable source for making definitive conclusions with reference to identifying a cause. Since most of the methods of sample collections were not standardised or statistically validated, making right interpretations and conclusions based on such data is also questionable. Most importantly, none of the postulated hypotheses explains the heterogeneity of the geographical distribution of patients with CKD-mfo.

\section{Prevalence of CKD-mfo and the demography of the affected people}

Many of those affected by CKD-mfo are young to middleaged male farmers who work on rice farms (paddy fields) and other agricultural commodity cultivations, where they presumably are exposed to unusually high levels of environmental hazards compared to their non-farming peers [27]. The affected occupations (predominantly paddy cultivation and some vegetable farming), male gender, and median age of 51 years when patients present with the disease, all point to occupational exposure (soil-water exposure and adverse environmental issues) contributing to causation of the disease [27].

In addition, within the region, the water quality varies markedly between villages, making reported water quality data difficult to interpret with reference to the disease pattern. Moreover, these data do not exclude certain potential causative factors. This heterogeneity may also reflect through the differences of prevalence of this disease between villages. 
It is estimated that in certain affected villages in the region, one in 10 or more adults experience renal impairment [6]. The overall prevalence (the mean) of those with all CKD in the region is about $12.5 \%$, but of those with CKD-mfo, it is approximately $7.5 \%$ [27]. The difference of $\sim 3 \%$ prevalence in the region may be attributable to other causes of CKD, such as long-standing diabetes and hypertension, snakebite, and so forth, that are unrelated to CKD-mfo. When one considers males who are older than 20 years, the prevalence of CKD-mfo is approximately $10 \%$. However, the final WHO report based on random sampling estimated an overall prevalence of CKD-mfo of more than $16 \%$ in the region [3], whereas others reported a prevalence of less than $3 \%$ [2]. It is difficult to explain this major discrepancy. Current data suggest that in the NCP, the overall prevalence of CKD-mfo is about $7.5 \%$ and perhaps as great as $13 \%$ to $15 \%$ amongst middle-aged men in certain regions [28].

Male farmers 25-65 years of age are the most affected and die of this disease in greatest numbers; they also frequently experience unprotected exposure to agrochemicals and other toxins in their harsh work environment, and also engage in high-risk behavior in comparison to women. On top of regularly getting dehydrated while at work and drinking less water, one of the plausible reasons male farmers are more exposed to potential toxins is that many have disregarded the age-old habit of taking clean water from home to the farmlands. They have adapted an easier and a dangerous habit of drinking polluted water directly from nearby streams and from the paddy fields, while women and children continue to drink water from relatively protected streams and wells near their homes.

While the local hospital morbidity and mortality statistics underestimate the disease prevalence, they support the finding that the disease incidence and deaths attributable to CKD-mfo are increasing each year. The current data and estimates suggest that the numbers of those with CKD-mfo are doubling approximately every 3-4 years. However, for multiple reasons, the overall statistics of those who are affected and the cause of deaths attributed to CKD-mfo are not reliable and are underestimated.

\section{Geographic distribution, characteristics, and potential clues of the origin of CKD-mfo}

One can learn from examining the characteristics of the disease pattern and the geographic distributions of this disease in Sri Lanka and other countries that are affected with CKDue. All CKDu-affected areas in the world are predominantly agricultural regions with agro-based economies; do not have centrally purified pipe bourn water supply, less than optimal access to medical care, and are
Fig. 1 a Geographical distribution of chronic kidney disease of multi-factorial origin (CKD-mfo) in Sri Lanka, illustrating the most affected areas; the North Central Province (NCP; illustrated in pink, red, and black) and the recent spread of the disease outside the NCP region (indicated in blue dots). b A map of Sri Lanka illustrating the distribution of groundwater hardness. The distribution of hard water, salinity, or ionicity does not correspond with the prevalence distribution of the CKD-mfo in Sri Lanka (lack of correlation, a, b). c A map illustrating the climatic zones of Sri Lanka: dry, intermediate, and wet zones. d A map illustrating the major rivers draining to the sea in Sri Lanka to illustrate their lack of relationship with CKD-mfo affected areas, except the River Mahaweli (indicated by blue arrows). The blue area indicates the most affected region, to which the River Mahaweli, the longest river in the country, supplies irrigated water

economically relatively poor. Those affected most are male farmers who work in harsh climatic conditions, in flat lands with poor drainage; they do not use protective gear when handling and spraying toxic agrochemicals, nor while working in farming land. These farmers frequently get dehydrated during working in hot, and harsh climatic conditions, and drink too little water (and some drink contaminated water directly from paddy fields). In addition, the affected areas experience unpredictable weather patterns with prolonged dry spells.

As with other CKDue affected countries, the disease in Sri Lanka is restricted to specific geographical terrains, in the drier zones (regions that receive precipitations during one monsoon period and drier conditions prevail rest of the year), including the NCP [2]. The CKD-affected areas cover approximately $24,000 \mathrm{~km}^{2}$ and include a population of approximately 3.0 million [1]. Of this, currently about 2.0 million are at high risk of contracting this deadly disease.

In Sri Lanka, approximately $90 \%$ of the affected families engage in paddy farming, whereas a considerable number also engage in vegetable farming or both. More than $80 \%$ of the affected people live in rural and remote communities, have little political power or voice, and have limited or no access to modern health care. Figure 1 shows maps of Sri Lanka with the geographical distribution of patients with CKD-mfo, the distribution of hard water, climatic zones, and the major rivers in the country. The River Mahaweli (indicated by arrows in Fig. 1d) irrigates the areas with the largest number of CKD-mfo affected patients.

This demographic situation in the affected areas is quite different from that of cities and suburban areas in the country, where most have access to a centrally purified, pipe-borne clean water supply and wholesome food, access to good health care, and education. The common variety of CKD affects females and males equally, mostly older people (median age of 64 years), and they generally have long-standing hypertension and/or diabetes; this is also true 

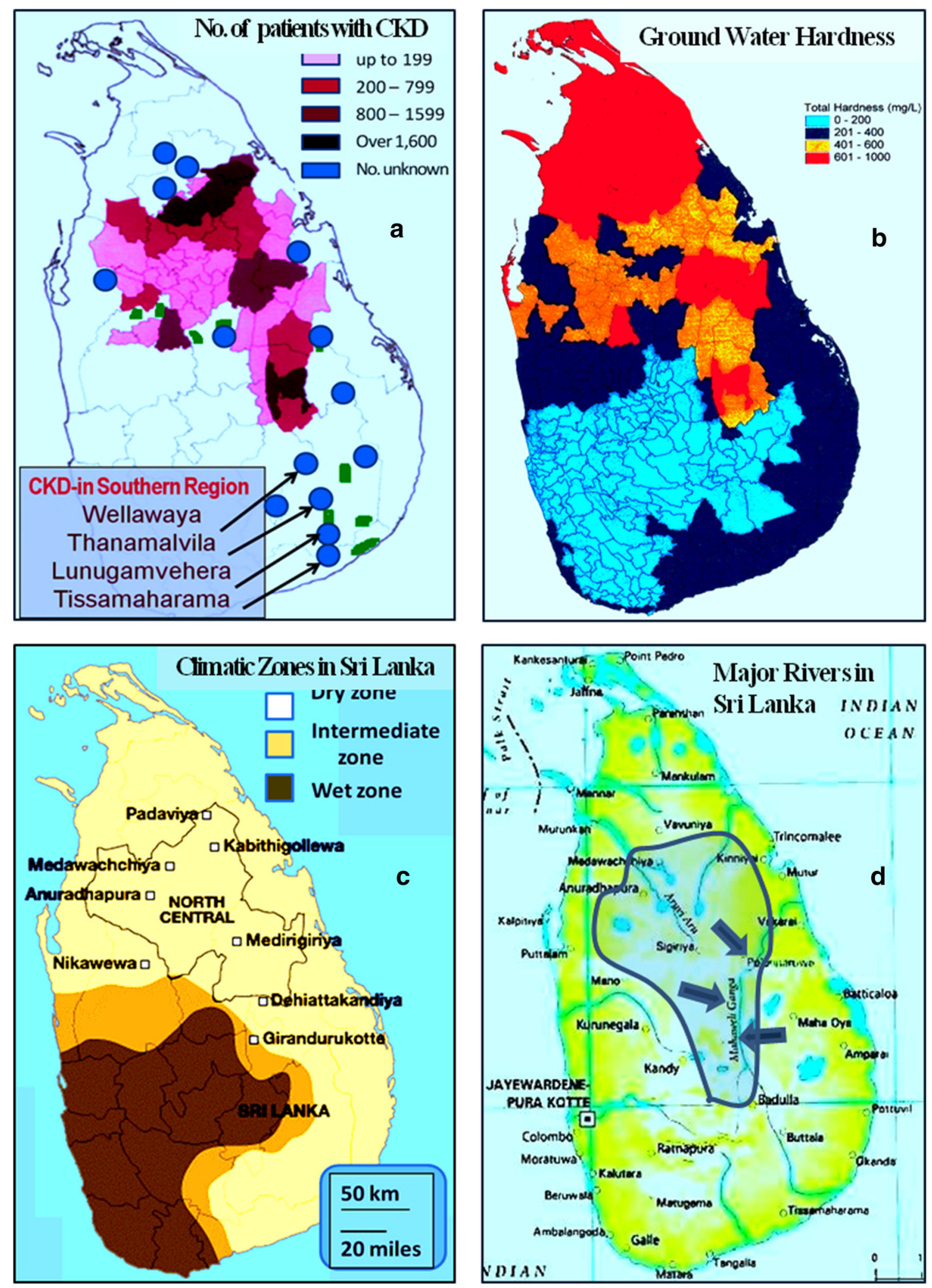
in the NCP. However, this pattern of CKD distribution is much different from those with CKD-mfo (mostly younger and middle age, with a median age of 51 years) in the NCP region [27].

\section{Distribution pattern of the disease}

The geographical distribution of patients of CKD-mfo is not uniform, and the prevalence is patchy and non-contiguous [5]; this highlights the importance of the geo-water contribution as a potential cause for the CKD-mfo. Taking into account the mosaic pattern of geographical distribution [2], an adverse soil-water, occupational environment exposure is likely to play a role in the CKD-mfo epidemic in Sri Lanka and other CKDuo affected counters. It is common to see villages with a high prevalence of CKD located within a few kilometres of villages without a high prevalence of the disease. Villages within the same region that have access to clean water springs (e.g. village, Kabithigollawa) or waterspouts have disease prevalence near zero. The anecdotal evidence suggests that over the past 3 years, the incidence of CKD has decreased by approximately $50 \%$ in a few villages where the National Water Supply and Drainage Board (NWS\&DB) has provided clean water. Another clue that contaminated water is a key source causing this disease.

On the other hand, the incidence of CKD-mfo is higher amongst those who consume water from shallow or dug wells, particularly the shallow wells located between paddy fields. Most of the drinking water wells are aquifer and regolith-fed through intergranular flow, and thus, water level fluctuates with cascade of reservoir water levels. The quality of well water varies considerably within one or two kilometres within the region. For example, there is a marked variability of natural fluoride content in water amongst villages. Therefore, making global conclusions based on water quality testing data taken from a relatively small number of water samples or scattered sampling can be misleading.

Naturally occurring and human-introduced trace elements display appreciable geographical variations [29]. The affected geographical region is large with relatively high usage of agrochemicals [27]. Nevertheless, the same agrochemicals are used in all other agricultural areas across Sri Lanka (in amounts, several times greater in certain regions, such as in Nuwara Eliya, where the prevalence of CKD-mfo is low or none), but higher rates of CKD-mfo are prevalent only in certain geographical regions in low land, dry zone region. In fact, five to ten times greater quantities of phosphate-rich fertilisers and pesticides are used in the Nuwara Eliya region in the hill country (i.e. higher elevation area; mountainous regions with high rainfall), where no case of CKD-mfo has been reported yet.
Therefore, factors other than agrochemicals are also likely to play a role in the occurrence of this disease. Moreover, no hypothesis put forward so far explains the multiple discrepancies, including the quantities of agrochemicals used, distribution of hard water and ionicity, eating or drinking habits, water contaminants, soil conditions, and the mosaic geographical distribution. These facts also indicate that more than one aetiological factor is responsible in precipitating CKD-mfo, and a multi-focal, broader approach is needed to identity root cause(s) and to alleviate the problem. Therefore, single-focus, narrowly designed, or laboratory-based studies are unlikely to be fruitful in identifying a cause of CKD-mfo, or find ways to prevent it.

\section{Postulations and hypotheses for the occurrence of CKD-mfo}

The groundwater tables in dry zones markedly fluctuate between the "wet" and "dry" seasons of the year. In the $\mathrm{NCP}$, the water table rises towards the end of the Northeast monsoon period, by early December. Thereafter it tends to fall gradually, reaching its lowest levels by early October. Many shallow wells go dry and the remaining well water is much concentrated with ions and dissolved particles. By about July/August, most of the shallow dug wells, the main source of drinking water for villagers in the NCP, are virtually empty. How this major fluctuation of water table and the changing water quality affecting human health is unknown. The only well characterised ailment is the higher incidence of renal stone formation and aggravation of existing kidney stone disease during the dry season.

The assorted patterns of the disease distribution strongly indicate that the environment and the geochemistry has an important impact on this condition [2,30]. Nevertheless, based on the demarcated geographical distribution of people with renal failure and CKD-associated deaths [5], groundwater testing data, and local farming habits [2], do not support any of the currently postulated components alone is likely to be the cause of the CKD-mfo in Sri Lanka. Multiple conflicting theories, correlations, and data sets have been reported [2-5, 17, 21, 31-39], yet none explains the geographic distribution pattern of the CKDmfo or identifies its cause. Therefore, before making conclusions, it is paramount to separate the reported correlations and hypotheses from causation of this disease [40].

Attempts made during the past decade to identify a cause for this CKD-mfo epidemic in Sri Lanka have failed to-date, in part because this illness likely is a multi-modal disease and studies conducted were not designed to cover broader concept(s). In addition, the study designs, data collections, and assumptions made by researchers varied 
Fig. 2 Multiple potential causes for CKD-mfo. Colour code: blue probable causes; yellow less probable causes; white causes that have not been investigated

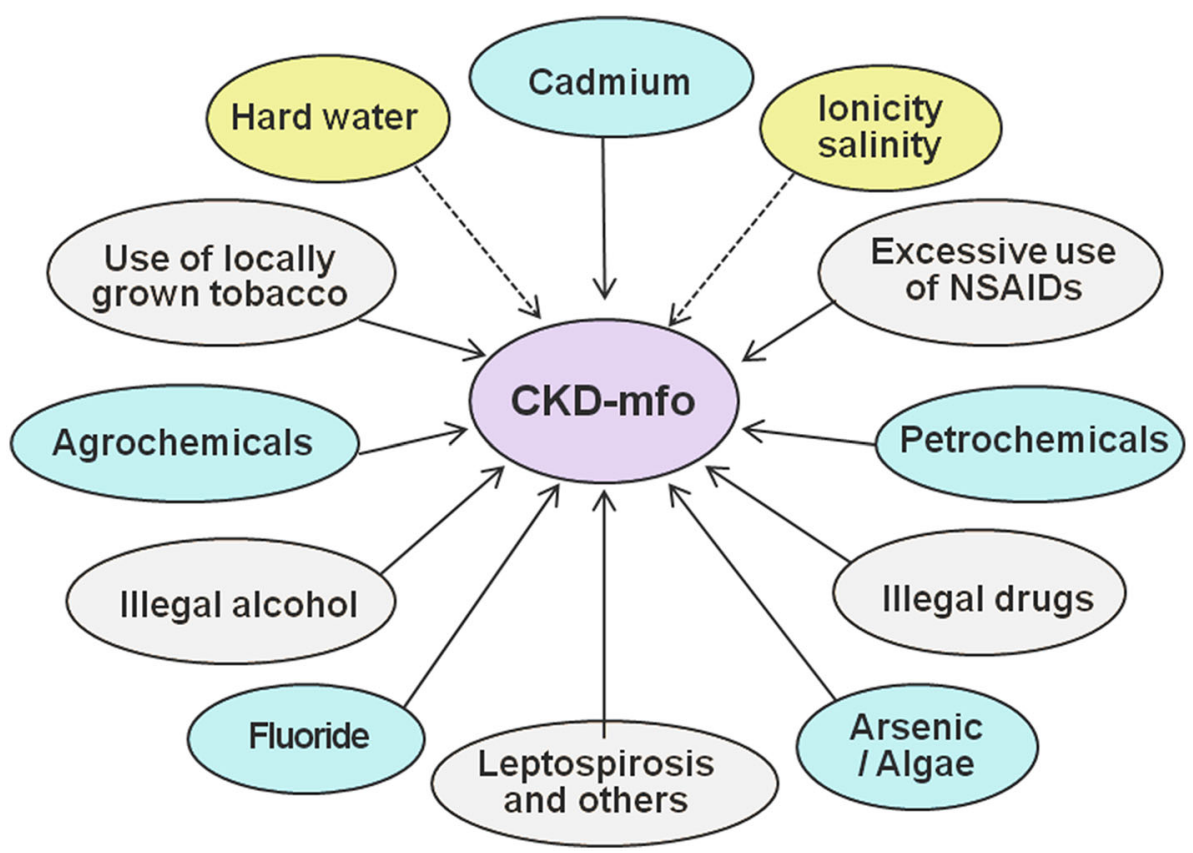

markedly. Therefore, none of the data sets can be pooled for meaningful scrutiny or accurate meta-analysis to draw conclusions. Narrowly constructed hypotheses and research directed to identify "one-cause" aetiology or 'prove' a hypothesis not only are likely to fail, but also may lead to incorrect conclusions.

Therefore, the continuation of research in the same line of thinking is unlikely to identify a cause(s) that precipitates CKD-mfo or present consequential and practical preventative strategies to eliminate the disease from the country. New ways of thinking and new direction for research are necessary. A summary of the key hypotheses put forward by scientists follows.

\section{Contamination from heavy metals}

Inorganic contaminants, including heavy metals, may be naturally occurring (e.g. arsenic in groundwater table) or may result from stormwater runoff, industrial or domestic wastewater discharge, gas and oil production, mining, or farming. Toxic heavy metals come from different sources, including poor quality chemical fertilisers (and arsenic and cadmium coming from the bedrock phosphate deposits such as in Eppawela), which continue to contaminate the farm soil and groundwater sources in agricultural regions in Sri Lanka and other developing countries. Tobacco that is grown on the embankments of the polluted Mahaweli River beyond Manampitiya contains higher-than-average amounts of both arsenic and cadmium [1]. Thus, the locally produced tobacco that is frequently smoked and chewed by farmers is another source of heavy metal intake, including arsenic and cadmium, which may enter the body via inhalation or the oral route.

Role of arsenic and cadmium

Arsenic is a ubiquitous element. Various researchers have reported that arsenic, cadmium, fluoride, and agrochemicals as potential causes of CKD-mfo in the NCP [4, 21]. High levels of arsenic consumption from contaminated food [41], water, and rice are a major concern in certain Asian countries [17, 42-44]. The accumulation of arsenic and cadmium in rice grains is influenced by water management and rice cultivation habits [45] that are commonly used in South Asian countries, but are not a significant issue in Sri Lanka [17, 46].

A report from the United States Food and Drug Administration indicates that the arsenic content of rice imported from India is twice as high as that of rice from Sri Lanka [46]. Another source of arsenic contamination is agrochemicals [47]; however, the reported arsenic levels in rice, water, and soil in the endemic dry zonal areas in Sri Lanka were not significantly different from those measured in the non-endemic, wet zone areas of Sri Lanka [2, 19, 33, 48]; thus, these findings do not support arsenic as a cause for CKD-mfo [3, 49].

Figure 2 illustrates different groups of chemicals and toxins that are postulated to cause CKD-mfo. One or more of these chemicals or toxins have been reported in water sources, soil, and commonly consumed food items, such as lotus (Nelumbo nucifera L.) rhizomes, rice, and tilapia fish in certain affected villages [4, 38, 50], but the levels are not high enough, and the finding is not uniform or common [3]. 
Moreover, the soil and water arsenic content varies greatly from region to region, yet the arsenic levels in rice kernels and soil show no significant differences in the endemic dry zonal areas and non-endemic wet zone of Sri Lanka [3, 19, 48]. In another set of studies, exposure to high levels of cadmium $(\mathrm{Cd})$ from reservoir water; irrigated rice; lotus rhizomes; freshwater fish, such as tilapia in the affected areas [4]; and milk from cows raised in cadmiumcontaminated pastures have been suggested as a link to CKD-mfo [4, 38, 50], but the data are not consistent, and other studies do not support this hypotheses [2, 27, 35].

Some researchers have suggested an association between the prevalence of CKD-mfo and the cascade irrigation systems [2], particularly the diverted water from the Mahaweli River [37] in Sri Lanka. However, other scientists have disputed this and failed to confirm these findings $[2,3]$. The contents of cadmium and other inorganic components in commonly used drinking water, freshwater fish, and rice from affected regions were in fact, lower [2, 3]. Nevertheless, the water diverted from the Mahaweli River is polluted with large quantities of washed-off agrochemicals and other materials coming from the hill country.

Most crops do not take up much arsenic from the ground, except paddy (rice) that takes up more arsenic from soil and water than do other grains [46]. Some seafood contains much higher levels of organic arsenic [51]. Nevertheless, the inorganic forms of arsenic are the ones that are associated with long-term ill health. Overall, reported data are non-conclusive with regard to any of the heavy metals under consideration, such as arsenic or cadmium, being a cause of CKD-mfo.

\section{Fluoride theory}

Both fluoride and arsenic are present naturally in the environment and water, and are normal groundwater contaminants [52]. Higher concentrations of fluoride are found in drinking water in certain shallow wells, but present in many of the deep tube wells that were tapped into a naturally high fluoride containing deep regolith/aquifer groundwater table. However, the distribution of fluoride in water is heterogeneous in the region [2, 52]. Exposure to high concentrations of fluoride ions $\left(\mathrm{F}^{-}\right)$for a prolonged period, however, can cause fluorosis [53] and kidney damage [51, 54].

Fluoride in combination with aluminium (Al) (such as from the substandard aluminium utensils used for boiling water and cooking) may produce alumino-fluoride complexes, which could become soluble and thus toxic, and contribute to the development or exacerbation of CKD-mfo [55]. These complexes may enhance the uptake of fluoride into the body because they can pass through biological membranes more easily than can fluoride and aluminium separately. Cooking in acidic media (e.g. vinegar) and during the process of making alcohol illegally can generate higher contents of aluminium fluoride complexes (so as solubilisation of other metal ions) that can leach out of these containers or from the contents into the cooking media, into food.

Some have suggested a possible correlation between the high concentrations of fluoride in drinking water and CKDmfo $[56,57]$. There are areas in Sri Lanka including Ampara, Pulmoddai, Monaragala and Wellawaya, with higher water fluoride contents but do not have a higher prevalence of CKD-mfo. But the disease seems to be slowly spreading to these area in recent years. However, the 2013 World Health Organization (WHO) group's report on CKD of unknown origin $(\mathrm{CKDu})$ in Sri Lanka dismissed this hypothesis [3]. The report rejected the association of fluoride with CKD-mfo on the basis that people have been consuming fluoride-containing water for generations in the region without ill effects. However, the report overlooked the gradual but substantial increase in the fluoride content in water in the region (particularly, following drilling hundreds of tube wells in the NCP region for drinking water), and the potential interactions amongst various key chemical components in the water that can be toxic to kidneys.

Because of a number of factors, including the ones mentioned above, during the last 20 years, the fluoride content and the extent of geographical distribution of fluoride in drinking water in the NCP region have increased. Some key reasons for this are the provision of a large number of deep tube wells intended to provide drinking water (water was tested only after a few years and found to have higher than the EPA stipulated amounts of fluoride), and the extensive evaporation of water from the water bodies in the region with a concentration of water secondary to prolonged droughts in the region and global warming. However, once the kidneys are damaged by one or more toxic components, as illustrated in Fig. 1 or some other unknown component, consumption of water containing higher amounts of fluoride can accelerate renal failure.

Other contaminants and toxins that have not been studied yet

Some of the other uncommon potential contaminants that can harm kidneys include organic chemicals, factory and vehicle discharges, by-products of industrial processing and petrochemicals/petroleum production (Fig. 2), coalburning power plants, gasoline stations, urban stormwater runoff, and discharges from septic systems. Meanwhile, the radioactive contaminants can be naturally occurring or the result of oil and gas production/exploration, hydraulic fracking, or secondary to mining activities $[51,58,59]$. 
Exposure to high concentrations of some of these chemicals and toxins (Fig. 2) and certain algae toxins [61] over a period of time can lead to glomerulo-tubular impairment and consequently urinary protein leak. Other implausible causes suggested include mycotoxins, ochratoxin A [31, 60, 61], hard water, and bioterrorism. Leptospirosis $[62,63]$ can cause renal damage and is widespread in the NCP and other affected regions in Sri Lanka. However, other areas in the country, particularly gemming areas such as Ratnapura, Balangoda, and Uggal-Kaltota, are heavily infected with leptospirosis but have few or no patients with CKD-mfo. In the presence of dehydration and other toxins, leptospirosis would enhance the liver and kidney damage [62, 64] (Fig. 2). Nevertheless, its role in the CKD-mfo in Sri Lanka has not been studied.

\section{Misuse of petrochemicals}

Cleaning agricultural equipment such as tractors in and around drinking water sources unfortunately has become a routine practice in the agricultural sector in many developing economies; the NCP region in Sri Lanka is no exception. There are no environmental regulations (or enforcements) for controlling such unthoughtful, manmade polluting practices, cleaning up petrochemical discharges and spills after the fact, or taking preventive actions. Therefore, these prevailing unhealthful practices continue to pollute water bodies, soil, and the environment, thus contributing to contamination of the food chain. These harmful practices and the lack of implementation of environmental regulations are likely to further aggravate CKDmfo and other non-communicable diseases in these economically poor, dry zonal agricultural communities.

\section{Genetic susceptibility hypothesis}

The reported clustering of the disease amongst smaller areas and within some families [17] does not necessarily indicate a genetic component for CKD-mfo, but more likely is attributable to exposure to a multitude of other factors, such as excessive exposure to agrochemicals in large quantities, unhealthy personal habits such as drinking from contaminated water supplies (usually from a common source), dangerous storage practices (storing toxic agrochemicals and household food items in the same location) and mishaps of toxic agents, indulgence of illegal, locally produced alcohol, and unforeseen interactions between fabricated and naturally occurring contaminants. The reported odds ratio of 2.13 for sodium-dependent dicarboxylate transporter-3 (which is a relatively common single-nucleotide polymorphism) is unlikely to be a causative aetiology for CKD; especially, half of the subjects in this particular study had additional non-communicable diseases. The latter further prevents meaningful data interpretation; making the genetic mutations or susceptibility theory less likely [33].

Agrochemical (pesticides and weedicides) contamination

Pesticides and herbicides may come from a variety of sources, including agricultural operations, urban stormwater runoff, and residential waste. Organophosphorus and other agrochemicals, particularly pesticides are toxic to humans and animals, whereas many herbicides, such as glyphosate, are harmful only to plants at the recommended doses [65-67]. The broadleaf herbicide propanil [N(3,4dichlorophenonyl)propanamide], the insecticide chlorpyrifos $\quad[O, O$-diethyl- $O$-(3,5,6-trichloro-2-pyridinyl)phosphorothioate], and the herbicide Roundup, which contains glyphosate [ $N$-(phosphonomethyl)glycine], are widely used in paddy and other commodity cultivations across the country and worldwide. However, none of the published data including the WHO report failed to identify detectable amounts of agrochemicals including glyphosate in water [3].

Long-term exposure to high concentrations of propanil, chlorpyrifos, and glyphosate may cause various chronic diseases, predominantly neurological disorders, endocrine disruptions, liver disease, and CKD [68-70], but the pattern of the renal disease following exposure to these is quite different from that of CKD-mfo. On the other hand, some of the recently introduced pesticides, such as neonicotinoids, incorporate into plants and crops and thus directly contaminate the food chain.

Nevertheless, there is no direct evidence linking these chemicals to CKD, and the available data are insufficient and inconclusive $[3,66]$. There is not a great deal of knowledge about the absorption of these components into the body via the oral route, but lipid-soluble compounds and chemicals can be absorbed through the skin or through inhalation via the pulmonary route. Few farmers in Sri Lanka use any protective apparatus, so skin and inhalation are potential routes of absorption of these toxic chemicals during their occupational exposure [27, 49]. Particularly worrying is that, farmers routinely spend in water-logged paddy fields with open feet, that further exposed them to dermal absorption of chemicals.

In addition, a number of insecticides and pesticides sold over the counter contain many other toxic ingredients. Some of these are discussed in the 2001 environmental treaty. The Stockholm Convention on Persistent Organic Pollutants [71] and are considered persistent organic pollutants (the "dirty dozen" of chemicals) [72]. Toxaphene $\left(\mathrm{C}_{10} \mathrm{H}_{10} \mathrm{Cl}_{8}\right)$ is one such insecticide [73]; after degradation, it releases free chlorine ions with the tendency to form 
chloroform (trichloromethane, $\mathrm{CHCl}_{3}$ ) in soil [74-76]. However, there is no evidence to suggest that the level of chloroform reached in soil or air is high enough to cause CKD-mfo.

Others have suggested that the occupational exposure to hydrocarbons may account for the excess number of male patients (male farmers) with CKDu [77]. Hydrocarbons are commonly used as solvents in manufacturing practices because of their lipid solubility [76]. Hydrocarbons are also known to be neurotoxic, and renal damage has been reported after ingestion [78]. In addition to the hepatic damage, hydrocarbons can cause renal tubular necrosis (interfering with the liver and renal cytochrome P450 enzyme systems) [77], but no data exist to indicate whether any of these cause the CKDu-like disease that is prevalent in the NCP $[79,80]$.

Because agrochemicals are generally considered toxic, it is not surprising that some of the commonly used agents have been associated with or linked to various human disorders [81-85], especially after longer term, high exposure. Researchers have shown some amounts of these agrochemicals to be present in water in small quantities (except phosphate in higher quantities in reservoir water) some of the affected areas $[4,30,68,69]$, but no data exist indicating that any of these chemicals directly causing CKD-mfo [8688]. In addition, these chemicals are used not only in the CKD-affected areas, but also throughout Sri Lanka and, in fact, globally. Therefore, none of these theories including the use of agrochemicals, explains why only certain geographically demarcated regions are affected with the disease, while farmers in other areas are not [49].

As described, to improve crop output and profitability, most farmers use increasing quantities of agrochemicals (in excess of the recommended amounts), such as pesticides, synthetic fertilisers, and in some cases plant growth-promoting compounds, to enhance yields [1,27]. Many of these frequently used agrochemicals and toxins are hazardous to human and animal health. Some of these agents could cause cancer [89], whereas others may lead to the development of genetic abnormalities, including gene mutations [90, 91]; negative reproductive effects; endocrine (hormone) abnormalities; and end-organ damage [89, 92]. CKD-mfo is an example of the latter, in which a combination of factors may lead to irreversible glomerulotubular kidney damage and renal failure.

\section{Effects of harmful agricultural and irrigation practices}

Hybrid varieties of imported seeds and breeds require higher amounts of water, and the crop output may vary depending on the use of agrochemicals and hormones; such varieties were introduced to Sri Lanka in the mid-1970s [93]. Plants became dependent on the use of chemicals, and

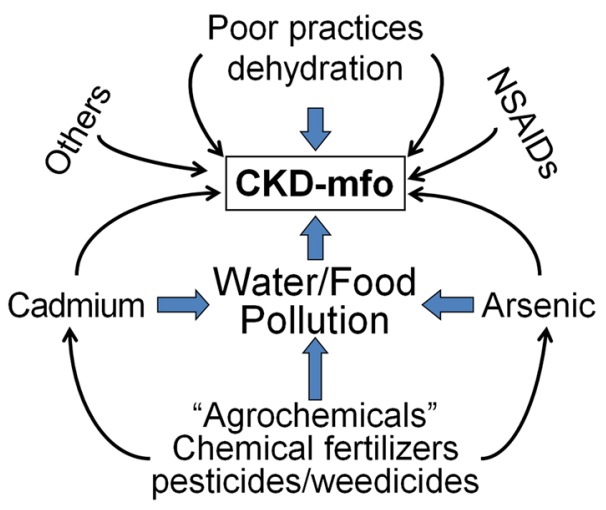

Fig. 3 Potential causes, their interactions, and the mechanisms explaining how the continual water and food pollution leading to the development of CKD-mfo among the farmers

farmers in turn became "hooked" on using such products in increasing quantities. The consequent enhanced crop yields discouraged the use of harmonious, traditional agricultural practices, leading to a marked increase in the use of and dependence on agrochemicals, imported into developing countries [94]; such importation also drains foreign exchange reserves in these developing economies. It is possible, but not proven that the over use of these agrochemicals and hormones may contribute to the development of CKD-mfo. In view of this, precautions and preventative actions need to be taken immediately to minimise the harm to farmers, fauna, flora, and the environment, and to protect consumers [95-98].

Traditional agriculture is characterised by ecologically sound, environmentally sustainable practices, such as crop rotation, inter-cropping, the use of animal manure and compost, and the use of natural pest-repellent substances; all of these methods avoid the use of toxic chemicals. However, farmers have acquired and are using certain unhealthy and harmful agricultural practices, including the unprotected handling and spraying of toxic chemicals, irresponsible use of agrochemicals, dumping of empty agrochemical containers and bags into streams, storing toxic chemicals with food, and discharging petrochemicals from agricultural equipment into waterways, and so forth. These practices harm not only the environment but also farmers themselves and their families. Many of these factors are interlinked, controllable, and thus not so difficult to overcome. Figure 3 illustrates various mechanisms and interactions, and the synergy amongst potential (preventable) causes of CKD-mfo.

\section{Negative effects of frequent dredging of canals and reservoirs}

Deforestation of slopes, particularly in the higher elevations, and the removal of the top-most fertile layer of soil 
by levelling the cultivation fields using bulldozers have become routine practices in developing countries, including Sri Lanka. This not only leads to water contamination through runoff and soil erosion, but also exposes the deeply deposited subsurface soils that may contain pollutants, particularly heavy metals $[1,2,99]$. In addition, these acts enhance the silting of reservoirs in the NCP region, requiring frequent de-sedimentation (i.e. taking mud and silt out of reservoirs) via dredging.

De-sedimentation is particularly concerning because many of the reservoirs that were built hundreds or thousands of years ago are now being dredged, exposing sediments containing toxins that have deeply settled onto the bottom of reservoirs. These materials have been settled in the bottom, undisturbed for centuries but now are pushed back into the water. These substances include heavy metals and other toxic compounds, are now contaminating the human food chain [20, 100]. Thus, the failure to adopt proper soil and water conservation measures throughout the country not only adds to water scarcity, but also to water contamination and human ill health, and among others, may contribute to the CKD-mfo epidemic.

In addition, farmers are using several times more than the recommended amounts of phosphate fertilisers, in part because they assume "more is better" and because of the large governmental subsidies that incentivised them to do so [27]. Moreover, once soil is excessively contaminated with phosphates, arsenic, or cadmium, it may take generations to rid the soil of these pollutants. The rates of pollution in part depend on dredging depth and its frequency, rainfall, and the water table. All planners and implementers must think seriously about the mistakes made in the recent past, particularly during the construction of new human settlements, dams and new irrigation systems; large-scale water diversions; and agricultural expansions. They must come up with practical ways to avoid repeating such expensive human sufferings and environmental errors in the future.

\section{The accelerated Mahaweli project, global warming, environmental changes, and the CKD-mfo}

Several major settlement or re-settlement projects were launched in Sri Lanka over the past five decades, particularly in the NCP and Eastern Province. These projects were implemented with little attention given to environmental or human safety issues. Therefore, it is not surprising, and in fact inevitable that negative consequences began to manifest years or even decades later [101]. In this regard, the Mahaweli River development and resettlement program in the dry zonal area in the NCP require special scrutiny [102-104] (The distribution of the River Mahaweli in

Table 1 Key causes that contribute to the development of CKD-mfo Item Potential causative factors References

A Careless use and overuse of pesticides, herbicides, and low-quality imported chemical fertilisers, which contaminate the environment with heavy metals and other toxic chemicals
B

Overuse of toxic agrochemicals, particularly chemical (phosphate) fertilisers as a result of continued government subsidy of these products

C Ineffective control of and lack of laws regarding the selling of these toxic products (e.g. virtually anyone can buy any quantity of these potentially dangerous chemicals over the counter) environmental damage and human adverse effects of chronic exposure to and irresponsible and overuse of agrochemicals

E Farmers in developing countries who handle and spray toxic agrochemicals do not use personal protective equipment, such as masks, gloves, goggles, footwear, and clothing. Thus, they are exposed to high concentrations of toxic agrochemicals (and petrochemicals) on a daily basis, which can get into the body via absorption through the skin or inhalation

F Lack of awareness leads farmers to carelessly overuse and continue to contaminate soil, water and the environment (e.g. disposing of used pesticide containers and bottles into streams)

G The use of unprotected (i.e. unlined) aluminium containers for cooking, which enhances solubilisation of aluminum fluoride, and thereby, increased the absorption and potential toxic effects of fluoride

$\mathrm{H} \quad$ Frequent dredging of canals and reservoirs reexposes deeply deposited toxins to water bodies and thus contaminates the food chain

I Consumption of fruits, vegetables, and grains sprayed before and immediately after harvesting with chemicals and preservatives, shining or waxing agents, including harmful carbofurans and antracols, and fungicides to serve as anti-transpirants

J Poor nutrition (e.g. lack of a balanced diet, which escalates chronic diseases, including CKD-mfo) promotes malnutrition

K Unintended long-term adverse effects from human settlement projects and large-scale irrigation projects, such as the accelerated Mahaweli project
D Lack of awareness by farmers of the

[106, 107]

$[1,108]$

$[83,109$,

$[27,111$, 112]

$[27,49$,

$[1,113]$

[116-119]

$[120,121]$

$[1,37,102$, 104]

relation to the CKD-mfo affected areas is illustrated in Fig. 1d).

In part because of political reasons [102], the proposed 30-year Mahaweli project was accelerated in 1977 (which also coincides with the duration of exposure to 
toxins that appear to be associated with the current CKD-mfo epidemic), to be completed in 6 years [102]. Since the initiation of this programme, which included newer fast-flowing hydraulic technologies, from an environmental point of view, things have worsened [37, 102, 104, 105].

It is plausible that this acceleration of irrigation project and human settlements have contributed to the current CKD-mfo epidemic [37], but there is no direct evidence. Nevertheless, this project negatively affected the fauna, flora, and human health in the region; the areas where water is now supplied by the diverted Mahaweli River water. In addition, the accelerated Mahaweli project also led to large-scale unintended re-exposure of deeply deposited heavy metals and other potential contaminants, which were returned to the dynamic water systems. With respect to human ill health, one potential example is the manifestation of CKD-mfo in these re-settlement areas (escalating incidence of Malaria is another example).

Those who are farming in the hill country, irresponsibly use larger quantities of agrochemicals, particularly phosphate fertilisers to enhance crops, much more than do those in the CKD-mfo affected areas, but the incidence of this disease in the hill country is low (or non-existent). Commonly used cultivations in this region like vegetables and potatoes can only absorb a fraction of these excess added fertiliser; the remainder wash away with rain. The lack of CKD-mfo patients in the hill country is explained by the unique terrain in the region, with its ubiquitous sloping landscape and high rainfall. With frequent rain, chemicals rapidly wash away with the surface water; these chemicals eventually end up in the NCP region via the Mahaweli River diversion. Some of the other broader possibilities that need to be considered as potential contributing causes of CKD-mfo are illustrated in Table 1.

\section{Importance of implementing natural, environmentally friendly agricultural methods}

In parallel with the preventative strategies mentioned, it is necessary to implement programmes to (a) generate naturally pest-resistant, high-yield crop varieties and select and propagate seed varieties that are not dependent on excessive amounts of water or synthetic agrochemicals; (b) reconstruct the ancient cascade irrigation system and tanks and restore the natural healthy human habilitations and environments around these reservoirs; (c) encourage farmers to decrease the use of synthetic agrochemicals and increase use of compost and other natural methods; (d) introduce environmentally friendly agricultural methods; (e) minimise environmental pollution; and (f) encourage soil and water conservation systems.
All of these should be part of the long-term strategy (food + agriculture + clean water + healthy environment $=$ good health). Water and the environment are national treasures. Therefore, it is important to educate and emphasise that all citizens have a duty to protect the country's water, air, and the environment. The governments must enforce environmental protection laws in the country without making any exceptions.

\section{The role of externalities in propagating CKD-mfo}

Each premature death affects not only the victim's family (including the loss-of-opportunity cost of dying prematurely), but also the entire community, taxpayers, and the economy of the country. Over the past five decades, numerous areas and practices in Sri Lanka have changed, including the Mahaweli River diversion, behaviour, eating and drinking habits, agricultural practices, socioeconomic diversity, and attitude to life and the environment, while various other new externalities have been introduced into the CKD-affected regions.

It is not just the agriculture or agrochemicals but likely a combination of many of these factors that contributes to the increasing incidence of non-communicable diseases and the current epidemic of CKD-mfo. Thus, re-labelling this disease as agricultural nephropathy or agrochemical nephropathy is inappropriate and, in fact, may be misleading. Farmers need to be educated to develop selfcontrol and self-discipline in the safe use of agrochemicals and to care about the safety of themselves and others, and clean potable water needs to be provided to not only to affected villages, but to the entire population in the region.

Steady economic growth and development require adherence to a long-term, coherent, national strategy. In the case of the CKD-mfo victims, the vast majority are male farmers; their agriculture and animal husbandry-based livelihood is threatened because of the epidemic of CKDmfo. Thus, it is essential that the government invest in the area of human capital now. Prevention of diseases such as CKD-mfo is easier and much more cost-effective than treating these later (i.e. using expensive renal dialysis, transplantation, etc.). Investing in the health and well-being of the populace will ensure better health and futures for all those living in the NCP and other CKD-affected areas, and minimise their voluntary migration (or a government initiated forced re-settlement later) into other regions, which would otherwise badly affect rice production and the economy in the country [27].

With the escalating incidence, doing little or nothing to prevent CKD-mfo and other escalating common noncommunicable diseases in the region is not an option anymore. However, because of the complexity of the 
overall situation, paternalistic approaches are unlikely to generate the intended healthy outcomes. Meanwhile, economic trade-offs may lead to unintended negative consequences. Investment today by the government is essential for future prosperity in the region and the entire country.

Agrichemical use and massive subsidies, expanding technology, unfair distribution of essential services and basic human needs, inequalities, and interfering politics are all externalities that have negatively contributed to changing the course of the health and well-being of the population, and the peace and prosperity of the NCP region and the country. These not only adversely influence health outcomes, but also drive costs for individuals and burden health care costs and the government. Nevertheless, some externalities require governmental intervention, such as negotiations, putting the correct policies together, health care and education, regulatory oversight, and enforcement of law and order. When implemented properly with longterm goals, right attitudes and good intentions, such interventions benefit the society.

On the other hand, improper behaviour, greed, creating pollution, and climatic changes all increase the personal, public, and governmental costs. Therefore, all citizens have a responsibility to minimise pollution and work towards a better future for generations to come. Moreover, studies have shown that the current choices and incentives rule the process and the future outcomes. Therefore, we need to provide people with basic safe amenities (such as access to potable water, safe sanitary facilities, affordable nutritious food, health care, and shelter), correct information and education, and then let the public decide which path to take. In this regard, the government's role should be to act as a referee, not as a player.

\section{Practical ways to curb the CKD-mfo epidemic}

There is no doubt that the provision of centrally purified pipe-borne water is the best solution, but at the current rate of investment on infrastructure, it may take five decades or more for such a system to materialise fully in Sri Lanka. However, an increased capital expenditure by an extra $10 \%$ per year, earmarked for infrastructure development in the affected regions by the NWS\&DB, can bring the timeline to approximately under two decades.

However, to be cost-effective, these funds must be dedicated to the development of a network of overhead storage tanks, pipelines, central water purification facilities, and other necessary infrastructure for the region in a transparent manner by the NWS\&DB. The supply of bottled water or daily transportation of water to the affected villages using bowsers by the NWS\&DB, are two of the most expensive, environmentally unfriendly, and unsustainable means for providing water to the region; thus, should be discouraged.

A broader project(s) should be encouraged and initiated through philanthropic organisations and with private-public partnerships to maximise the human and material resources, minimise governmental bureaucracy and duplications, and generate the funds needed to address this vital issue and save lives in a shorter period. Charitable organizations can handle these areas more efficiently and costeffectively than any governmental body. This would also allow the NWS\&DB to focus on its competence; the infrastructure development, instead of diverting its attention to the installation of reverse osmosis water purification plants. The latter would more cost-effectively and efficiently can be handled by philanthropic organisations. This would also, allow the NWS\&DB to expedite construction and delivery of a central systems that provides purified clean water to the entire region in a long term sustainable manner [122].

Performing such work though a unified philanthropic organisation has many advantages, including bringing expatriates, interested local groups, and resources together, facilitating and coordinating actions on a clear and focussed path, combining public education programmes with unified clear messages, taking care of families destitute by premature deaths of the breadwinners, and providing safe and clean drinking water to all affected areas and neighbouring villages at an affordable cost, in the least amount of time [1, 122] (https://wimalawansa.org/phepro).

In addition, while avoiding duplications, efforts should focus on the matter at hand and offer what is essential for the villagers to decrease the incidence of CKD-mfo as earliest possible. If the mentioned broader programme is implemented, it is estimated that the occurrence of new cases of CKD-mfo in the region will start to decrease markedly within 2 years, halving the incidence in four to five years, with the possibility of eradicating CKD-mfo from Sri Lanka within 15-20 years. However, the set goals will not be achievable by just providing clean water alone without attending to the prevention of environmental pollution in the entire region. To keep the population healthy and productive, and prosperous, these policies should be applied to the entire country.

\section{Recommendations for reducing the incidence of CKD- mfo}

The government and the Department of Health have an ethical and fiduciary responsibility to provide the necessary treatments for patients with CKD and related issues. This responsibility includes, but not limited to, providing access to care and medicine, adequate dialysis facilities, 
transportation of patients to these centres and arrange overnight stays for patients; and making dialysis and medications affordable. A portion of the massive governmental subsidies spent on agrochemicals should be transferred to taking care of affected people and prevention of this deadly disease. Nevertheless, since the funds are limited, government and charity alike should use their funds wisely to prevent people from getting this deadly disease, rather than spending on theoretical research or propaganda with no benefit to the NCP community or expanding hospital-based dialysis centres; neither of these would have any effect on preventing people getting this deadly disease.

Taking firm actions to prevent people from contracting the disease would not only be cost-effective, but also would decrease the incidence and prevalence of CKD-mfo, and reduce the need for expensive dialysis centres. In addition, those with early stages of renal failure need to be identified as early as possible and treated efficiently (including the provision of clean water and encouraging them to consume it regularly) to potentially reverse the disease process at early stages [i.e. preventing the disease progressing to CKD stage IV (renal fibrosis) and beyond]. Anecdotal evidence suggests that the regular consumption of clean water by people affected with early stage CKD together with proper nutrition, other basic medications, and vitamin $\mathrm{D}$ adequacy may reverse early stages of renal failure, before kidneys begin to fibrose.

Most patients who contract CKD-mfo are going to require renal dialysis as a life-saving mechanism. However, the vast majority of patients with CKD-mfo in the region are poor and cannot afford to travel to cities several times a week for dialysis or even to purchase essential daily medications. Neither, the vast majority of them cannot find suitable kidney donors nor able to afford renal transplantation costs. Thus, prevention is the key to curbing the epidemic of CKD-mfo in Sri Lanka [1]. It is achievable by a combination of various initiatives discussed here and presented previously by the author at various venues $[1$, 22].

In addition, considering the increasing numbers of families falling into destitution because of the demise of their breadwinners, it is necessary to establish a NCP-wide welfare programme to support women and children (particularly to maintain children's nutrition and education) in affected families. However, implementation of an effective CKD-mfo prevention programme in the region would decrease the longer term need for such a welfare programme.

The excess and irresponsible use of fertiliser is likely to contribute to the development of this disease. Therefore, it is necessary to reduce fertiliser consumption back to the levels recommended by manufacturers and the Department of Agriculture. The most efficient way to decrease the use of phosphate fertilisers in the country is to release it to famers based on judicious soil analysis data. Since there is no existing infrastructure to do this, it requires setting up of a new institution for nationwide soil testing together with water analysis, as well as for analysis of fertiliser components and pesticides, and an independent "department of toxic substances control."

This new institute dedicated for soil analysis and water testing must be committed to the goals and uncorrupted, and should be present in all agricultural regions in the country providing its services for soil and water analysis. This is a service that should be offered to farmers with a subsidy. Issuing of fertiliser to farmers based on soil analysis data alone could reduce the current excessive and wasteful fertiliser consumption by approximately $10 \%$ a year, a major savings for the hosting government in subsidy costs alone. In Sri Lanka, this action alone would amount to about 4 billion rupees per year; savings from the fertiliser subsidy and expenses. This is in fact more than double of what is needed yearly as the expenses for the prevention and eradication of CKD-mfo in the country. If implemented properly, no extra or outside funds necessary to prevent and eradicate CKD-mfo from the country. Using these savings, an additional novel subsidy-fund also could be created for farmers to purchase ploughing equipments that would indirectly decrease the need of using herbicides.

In addition to false expectations and greed [27], the main reason for overuse of fertiliser by farmers is that these commodities are highly subsidised [1, 27]. Consequently, most farmers indiscriminately use fertiliser aggravating environmental contamination [27]. Therefore, although it may not be palatable with the farmers, it is necessary for the government to reduce agro-subsidies as a disincentive to preventing such unproductive behaviour. As mentioned above, this can be achieved by (a) issuing fertiliser based on local soil testing data and/or (b) directly decreasing the governmental subsidy by $10 \%$ a year from the source [49]. In addition to tighter control of agrochemical importation, strict quality control systems for imported and locally manufactured agrochemicals, and controlled retailing to local farmers, it is essential to educate and encourage farmers on the proper handling and the use of agrochemicals and protecting themselves and the environment.

On the prevention front, it is necessary to execute a regional and a countrywide programme to educate farmers and villagers, using the mass media on regular basis, and compatriots regarding the importance of prevention of water and environmental pollution and the use of personal protection when handling harmful chemicals. This should include ways to prevent water contamination and education on the consequences of consuming contaminated water, providing farming communities with information on the use of personal protective equipment, and making those 
devices available at an affordable cost. It would be wise to get the national and locally represented multi-national agrochemical industries to implement the latter recommendation, under the supervision of the expertise of the Department of Agriculture.

In parallel, it is critical to provide safe and clean potable water to all affected areas, earliest possible. Implementing these measures would lead to a decrease in the incidence of CKD-mfo (i.e. reducing the appearance of new cases) and stabilise or even improve the conditions of those with early-stage renal disease.

The Department of Agriculture and the Environmental Protection Authority has profound fiduciary responsibilities for protecting the water and environment. They must educate farmers and others on the importance of and ways to prevent pollution, and encourage natural and environmentally friendly, sustainable farming methods. They also need to arrange collection and safe disposal of used agrochemical bags and containers, teaching the proper and responsible use of agrochemicals and the disposal of empty containers; ensure the safety and quality of all local and imported agrochemicals; and control the sale of such products.

The Environmental Protection Authority in particular should take all efforts to enforce environmental protection laws. To eradicate CKD-mfo successfully, all governmental agencies must work together synergistically and programmes need to deploy promptly in "parallel" with a countrywide surveillance programme for detecting new trends in CKD-mfo. The government should also consider promoting mechanisms, provide incentives for privatepublic partnerships, encourage and nuture philanthropic organisations to widen the opportunities for assisting affected people, and synergize such efforts.

To overcome the CKD menace and prevent spreading the disease into other areas (see Fig. 1a), the best options are, (A) to educate farmers on the prevention of water and environmental contamination and (B) provide them with constant, easy, and affordable access to safe and clean potable water. It is imperative that these two measures be done immediately and effectively, in parallel with achieving the goal of decreasing the incidence and eventually eliminating CKD-mfo from the region and the country. Public funds that saved from reducing agro-subsidies should be released to cover these focussed and effective activities. All required funds can be generated within, by reducing the vast and wasteful expenditures on agrochemical subsidies.

\section{Discussion}

Although the exact cause of CKD-mfo is not identified, it most likely is attributable to a combination of factors, as illustrated in Figs. 2 and 3. CKD-mfo is a major socioeconomic and health problem and is likely to become a significant political problem in the near future in Sri Lanka. The prevalence of CKD-mfo has increased strikingly during the past 20 years [17], doubling the incidence every 3-4 years, and currently affecting more than 100,000 people. This deadly disease is negatively affecting the livelihoods of thousands of farming families and consequently, the economy of the region and the entire country [123-125]. Water pollution goes together with the contamination of the food chain; thus, the two cannot be separated. Once the chemicals and toxins get into the human food chain, long-term disastrous health consequences are inevitable, and a negative vicious cycle is established. One of the goals should be to break this negative vicious cycle and re-establish the healthy environment, food-chain and health.

Traditional agriculture was used for generations in Sri Lanka and in other agricultural societies in the world. Organic agriculture is a farming method that does not use synthetic agrochemicals or plant/animal hormones; it is currently used only by approximately $8 \%$ of farmers worldwide. In fact, a meaningful recycling process (rather than burning material), similar to what naturally occurs in forests in harmony with nature, should be encouraged in all farming fields.

Such methods naturally recycle plant and animal material in the ecosystem in a sustainable manner without the intervention of humans, just as occurs in jungles and in coral reefs in the absence of human intervention. This natural recycling provides harmonious supplies of balanced plant and animal nutrients for a complete, healthy and sustainable ecosystem. Unfortunately human interventions are constantly disrupting this natural cycle. This mismatch in energy flow leads to ecological imbalances, among other, influencing destructive climatic change (droughts, floods, forest fires, and hurricanes), exposure of toxic elements, emergence of new diseases, and so forth.

Recent reports, including the conclusions of the WHO group's CKDu report, provided inconclusive results [3]. In Sri Lanka, many of the potential single causes that have been postulated and studied have been found not to be causative for (but may be associated with) CKD-mfo. Nevertheless, multiple renal toxins, even when present at lower than the renal threshold of individual components $\left(\mathrm{LD}_{50}\right)$, can cause renal damage (i.e. additive or synergistic pathological effects in kidneys), especially if they have different modes of actions causing renal tubular damage.

With reference to the identification of a causative factor, moving directly from "hypothesis" to "conclusions," as has been done in many CKDu-affected countries, including El Salvador, La Sale, Nicaragua, and Sri Lanka, is unscientific and is an important barrier to progress. Such 
approaches, which bypass important scientific steps and processes, including theory and experiments, prevent making firm and practical conclusions, further delaying identification of causative agent(s) of CKD-mfo. Although individual scientists have done outstanding work, all studies to date were based on narrow or single-cause hypotheses; as noted, because of the potential interactions of various agents, such approaches are unlikely to reveal true causative factor(s). Because of the lack of coordination and open dialogue amongst scientists, an effort is needed to standardise research protocols and provide results for public access, such as in a registry or repository to make the data coherent, available, useful, and easier to interpret.

Because CKD-mfo is likely to be caused by adverse alterations in the soil-water environment, there is no medical solution to eliminating it. Instead, research, social and educational solutions need to be directed to broader aspects of "preventing CKD-mfo." These include costeffective ways of cleaning up of contaminated soil, water and the environment, novel effective methods of environmental preservation and public education strategies, development of novel hybrid insect resistant seed varieties that require less agrochemicals and water, development of novel engineering methods to harmonize agricultural and human activities, provision of clean water to everyone in the affected areas, and other effective means to prevent people contracting this deadly disease.

Over the past decade, the research funded by the national science organisations has failed to establish an effective strategy for preventing CKD-mfo. Therefore, to make an impact on this disease, the direction of the research carried out with public funds in the country must be channeled to "prevention" of this disease. To accomplish this goal, it is highly advisable that all research funds to be directed via a dedicated body consisting of qualified senior scientists (preferably retired scientists to avoid conflicts of interests), who function independently from ministries, departments and government-sponsored science organisations to identify research priorities and direct to all CKD research in the country.

\section{Conclusions}

The current epidemic of CKD-mfo is causing a large-scale human suffering and economic hardship to victims and their families, similar to the harm done by other common diseases induced by environmental factors, such as cigarette smoking, alcohol, and exposure to asbestos. The solution does not lie in the expansion of dialysis centres but in promptly intervening with effective programmes to prevent this disease. When one person in a family is chronically ill, the entire family is burdened; the effects are particularly devastating for economically deprived families, which include the vast majority of families in the NCP and other affected regions in the country.

Not only CKD-mfo leads to end-stage renal failure, but also has major consequences for affected individuals and their families, especially a major economic and social burden, and a social stigma [126], and constrains the limited health care resources. Although the excessive use of illegally produced alcohol, cigarettes, and certain medications negatively affects renal function, a balanced diet, especially the intake of fruits and vegetables, anti-oxidants, and an adequate amount of vitamin $\mathrm{D}$, and drinking plenty of clean potable water likely to protect kidneys [127, 128].

An association was also reported between micro-albuminuria and consumption of potentially contaminated well water in the NCP region, which further suggests an environmental aetiology for CKD-mfo in NCP [4]. However, having protein in urine (random sampling) is not diagnostic of having CKD-mfo. For example, hypertension, diabetes mellitus, and smoking, or fever due to any reason, or contamination of urine during its collections are known to falsely diagnosed having micro-albuminuria; a marker that is considered for renal impairment $[4,5]$. Considering the above-mentioned, detecting micro albuminuria does not diagnose renal impairment or CKD-mfo, as there could be many false positives. As a result, CKD-mfo is incorrectly (falsely) diagnosed in many patients in the region (by simply detecting the presence of small quantities of protein in random urine samples, that was not repeated; these are false-positive diagnoses). These are the patients said to be easily "cured" using various native methodologies, but they probably never had renal failure to start with. In patients with CKD stages beyond III, their kidneys are irreversibly damaged.

According to the Bradford Hill criteria of causality of diseases [129], none of the research reported to-date provides direct evidence of causality of CKD-mfo attributable to any of the postulated suggestions as illustrated in the Fig. 2. In view of the escalating incidence of CKD-mfo, the enormous drain on resources of the health system, and the loss of productivity that negatively affecting individual families and the economy, immediate actions to resolve this issue are needed; this must be considered a national priority in Sri Lanka and other CKDu-affected countries.

Similar action plans are appropriate for all countries that are affected by CKD-mfo/CKDu. In addition to commencing a well-designed surveillance programme [123, 124, 130], Sri Lanka must conduct a prospective, adequately statistically powered (or statistically well designed cluster sampling), region-wide, collaborative, multi-disciplinary, long-term, well-designed epidemiological scientific study to reveal the root causes or causative factors of CKD-mfo and identify spreading patterns, which would 
facilitate the process of eradication of the disease. However, this should be done in parallel with strenuous prevention efforts with all governmental departments and ministries, and philanthropic organizations are working together.

CKD-mfo is an environmentally acquired occupational disease. It did not arise out of one isolated incident, terrorism, Mahaweli river diversion, or because of irresponsible use of agrochemicals alone, but can be attributed to the imbalanced and unsustainable social and economic developments, an alienated agricultural practices, that have been implemented in the country since independence, particularly during the past four decades.

The government, Department of Health, NWS\&DB, Environmental Protection Authority, and the Department of Agriculture, all must work together in a coordinated manner with philanthropic organisations and the private sector to synergistically implement an effective long-term programme to prevent the occurrence of CKD-mfo and other chronic non-communicable diseases in the country. However, despite the escalating incidence of CKD-mfo, there has been a considerable delay in taking preventative actions.

CKD-mfo is an environmental pollution-related occupationally-acquired disease [131]. No matter how much one would spend, there is no specific medical intervention one could provide to prevent or cure CKD-mfo; thus, prevention is the best way ahead. Public funds should not be targeted on curative health and related research, but directed to enhance preventive health programmes, provide potable water, education of farming families, clean up the environment, and provide enhanced environmental protection [131].

Provision of clean potable water, broader education, prevention of pollution, and long-term environmental preservation efforts are the ways ahead to decrease the incidence of CKD-mfo. Creating multiple Task Forces would not have any meaningful impact on preventing and eliminating this deadly disease from Sri Lanka. On the other hand, the prevention of the escalating incidence of CKD-mfo in Sri Lanka and other affected countries requires (A) governmental dedication and commitment, the establishment and funding of a new entity, "CKDAlleviation Authority," which needs to be fully independent of ministries and government departments, (B) the introduction and enforcement of legislation on pollution prevention and control, (C) development and incorporation of sustainable agricultural methods, and (D) the provision of safe and clean drinking water and safe sanitary facilities to all communities. Delays in the provision of effective preventative solutions in the $\mathrm{NCP}$ and other affected regions in Sri Lanka would increase sickness and CKD-associated premature deaths and contribute to the economic downturn of the entire country. However, prompt and right actions can prevent this disaster.

Acknowledgments The author is grateful for the constructive suggestions of Dr. Manjula Bandara and Ms. Geethanjali Selvendran. For the past 15 years, the author has been studying issues related to water contamination, escalating chronic diseases, and the provision of education and clean water to prevent the propagation of CKD-mfo in Sri Lanka.

Conflict of interest The author has no conflict of interest.

\section{References}

1. Wimalawansa SJ. Water pollution-associated ill health: special emphasis on chronic kidney disease in Sri Lanka. https://wima lawansa.org/sites/all/files/Olcott_Oration_2013_b.pdf, http:// www.anandacollege.net/document/14011809Olcott\%20Ora tion\%20Book.pdf. Olcott Memorial Oration. 2013, Ananda Collage: Colombo, Sri Lanka, p. 1-12.

2. Chandrajith R, et al. Chronic kidney diseases of uncertain etiology (CKDue) in Sri Lanka: geographic distribution and environmental implications. Environ Geochem Health. 2011;33(3):267-78.

3. WHO-Group, Jayathilaka N, Mendis S, Mehta FR, Dissanayake LJ, Janakan N. Investigation and evaluation of chronic kidney disease of uncertain aetiology in Sri Lanka (final report), WH Organization editor. 2013, Colombo, Sri Lanka.

4. Wanigasuriya KP, Peiris-John RJ, Wickremasinghe R. Chronic kidney disease of unknown aetiology in Sri Lanka: is cadmium a likely cause? BMC Nephrol. 2011;12:32.

5. Wijewickrama ES, et al. Epidemiology of chronic kidney disease in a Sri Lankan population: experience of a tertiary care center. Saudi J Kidney Dis Transpl. 2011;22(6):1289-93.

6. Athuraliya NT, et al. Uncertain etiologies of proteinuric-chronic kidney disease in rural Sri Lanka. Kidney Int. 2011;80(11): 1212-21.

7. Rajapurkar MM, et al. What do we know about chronic kidney disease in India: first report of the Indian CKD registry. BMC Nephrol. 2012;13:10.

8. Zhang J, et al. Association between aristolochic acid and CKD: a cross-sectional survey in China. Am $J$ Kidney Dis. 2013;61(6):918-22.

9. Ramirez-Rubio O, et al. Chronic kidney disease in Nicaragua: a qualitative analysis of semi-structured interviews with physicians and pharmacists. BMC Public Health. 2013;13:350.

10. Voice TC, et al. Evaluation of the hypothesis that Balkan endemic nephropathy is caused by drinking water exposure to contaminants leaching from Pliocene coal deposits. J Expo Sci Environ Epidemiol. 2006;16(6):515-24.

11. Stefanovic V, et al. Fifty years of Balkan endemic nephropathy: challenges of study using epidemiological method. Ren Fail. 2009;31(5):409-18.

12. Martins D, Agodoa L, Norris K. Kidney disease in disadvantaged populations. Int J Nephrol. 2012;2012:469265.

13. Parameswaran S, et al. Referral pattern of patients with endstage renal disease at a public sector hospital and its impact on outcome. Natl Med J India. 2011;24(4):208-13.

14. Sumaili EK, et al. Epidemiology of chronic kidney disease in the Democratic Republic of Congo: review of cross-sectional studies from Kinshasa, the capital. Nephrol Ther. 2010;6(4): 232-9. 
15. Markovic B. Balkan nephropathy and urothelial cancer. Well water pollution and current questions on pathogenesis and carcinogenesis. Prog Urol. 1993;3(1):98-107.

16. Reddy DV, Gunasekar A. Chronic kidney disease in two coastal districts of Andhra Pradesh, India: role of drinking water. Environ Geochem Health. 2013;35(4):439-54.

17. Nanayakkara $S$, et al. Evidence of tubular damage in the very early stage of chronic kidney disease of uncertain etiology in the North Central Province of Sri Lanka: a cross-sectional study. Environ Health Prev Med. 2012;17(2):109-17.

18. Senevirathna L, et al. Risk factors associated with disease progression and mortality in chronic kidney disease of uncertain etiology: a cohort study in Medawachchiya, Sri Lanka. Environ Health Prev Med. 2012;17(3):191-8.

19. Marmot M. Social determinants of health. Oxford: Oxford University Press; 1999.

20. Rathnamalala NK, et al. Patient perceptions of risk factors for chronic kidney disease and methods of delaying progression of the disease in a tertiary care setting in Sri Lanka. Int J Clin Pract. 2011;65(10):1108.

21. Wickremasinghe AR, Peiris-John RJ, Wanigasuriya KP. Chronic kidney disease of unknown aetiology in the North Central Province of Sri Lanka: trying to unravel the mystery. Ceylon Med J. 2011;56(4):143-6.

22. Wimalawansa SJ. Water pollution and chronic kidney disease in Sri Lanka. 2014. http://www.dailymirror.lk/opinion/172-opi nion/43526-water-pollution-and-chronic-kidney-disease-in-srilanka.html. Accessed Feb 2014.

23. Herath HM, et al. Chronic kidney disease in snake envenomed patients with acute kidney injury in Sri Lanka: a descriptive study. Postgrad Med J. 2012;1037(88):138-42.

24. Wanigasuriya KP, et al. Chronic renal failure in North Central Province of Sri Lanka: an environmentally induced disease. Trans R Soc Trop Med Hyg. 2007;101(10):1013-7.

25. Souqiyyeh MZ, Shaheen FA. Survey of attitudes of physicians toward the current evaluation and treatment of chronic kidney disease-mineral and bone disorder (CKD-MBD). Saudi J Kidney Dis Transpl. 2010;21(1):93-101.

26. Ito H, Kinugasa E. Pathogenesis of secondary hyperparathyroidism and renal bone disease. Clin Calcium. 2004;14(5): $720-5$.

27. Wimalawansa SA, Wimalawansa SJ. Impact of changing agricultural practices on human health: chronic kidney disease of multi-factorial origin in Sri Lanka. Wudpecker J Agric Res. 2014;3(5):110-24.

28. Wimalawansa SA, Wimalawansa SJ. Escalating incidence of chronic kidney disease of multi-factorial-origin (CKD-mfo) in Sri Lanka and its long-term consequences. Int J Pub Health Res 2014b (submitted).

29. Winkel LH. Environmental selenium research: from microscopic processes to global understanding. Environ Sci Technol. 2012;46(2):571-9.

30. Baumgarten A, Steinnes E, Friesl-Hanl W. This special issue of "environmental geochemistry and health", compiles the output of the symposium "soils and their implication on health". Preface Environ Geochem Health. 2009;31(5):521-2.

31. Wanigasuriya KP, et al. Could ochratoxin A in food commodities be the cause of chronic kidney disease in Sri Lanka? Trans R Soc Trop Med Hyg. 2008;102(7):726-8.

32. Wimalasurendre C, Amarasiri S. Scientists warn of time bomb: Phosphate and algae boom in reservoirs in Sri Lanka, 2013. http://www.island.lk/index.php?page_cat=article-details\&page $=$ article-details\&code_title=90856. Accessed 4 June 2014.

33. Nanayakkara S, et al. An integrative study of the genetic, social and environmental determinants of chronic kidney disease characterized by tubulointerstitial damages in the North Central Region of Sri Lanka. J Occup Health. 2014;56(1):28-38.

34. Jayasumana C, Gunatilake S, Senanayake P. Glyphosate, hard water and nephrotoxic metals: are they the culprits behind the epidemic of chronic kidney disease of unknown etiology in Sri Lanka? Int J Environ Res Public Health. 2014;11(2):2125-47.

35. Chandrajith $\mathrm{R}$, et al. Dose-dependent $\mathrm{Na}$ and $\mathrm{Ca}$ in fluoride-rich drinking water-another major cause of chronic renal failure in tropical arid regions. Sci Total Environ. 2011;409(4):671-5.

36. Bandara JM, et al. Chronic renal failure in Sri Lanka caused by elevated dietary cadmium: Trojan horse of the green revolution. Toxicol Lett. 2010;198(1):33-9.

37. Bandara JM, et al. Pollution of River Mahaweli and farmlands under irrigation by cadmium from agricultural inputs leading to a chronic renal failure epidemic among farmers in NCP, Sri Lanka. Environ Geochem Health. 2011;33(5):439-53.

38. Bandara JM, et al. Chronic renal failure among farm families in cascade irrigation systems in Sri Lanka associated with elevated dietary cadmium levels in rice and freshwater fish (Tilapia). Environ Geochem Health. 2008;30(5):465-78.

39. Athuraliya TN, et al. Prevalence of chronic kidney disease in two tertiary care hospitals: high proportion of cases with uncertain aetiology. Ceylon Med J. 2009;54(1):23-5.

40. Hill AB. The environment and disease: association or causation? Proc R Soc Med. 1965;58:295-300.

41. Wang HS, et al. Arsenic concentration in rice, fish, meat and vegetables in Cambodia: a preliminary risk assessment. Environ Geochem Health. 2013;35(6):745-55.

42. O'Neill A, et al. Arsenic in groundwater and its influence on exposure risks through traditionally cooked rice in Prey Veng Province, Cambodia. J Hazard Mater. 2013;2562:1072-9.

43. Hite AH. Arsenic and rice: a call for regulation. Nutrition. 2013;29(1):353-4.

44. Wong WW, et al. Dietary exposure to inorganic arsenic of the Hong Kong population: results of the first Hong Kong total diet study. Food Chem Toxicol. 2013;51:379-85.

45. Hu P, et al. Water management affects arsenic and cadmium accumulation in different rice cultivars. Environ Geochem Health. 2013;35(6):767-78.

46. Food and Drug Administration USA, Arsenic in rice and rice products (FDA web site). 2013. http://www.fda.gov/Food/Food borneIllnessContaminants/Metals/ucm319948.htm

47. Halder D, et al. Risk of arsenic exposure from drinking water and dietary components: implications for risk management in rural Bengal. Environ Sci Technol. 2013;47(2):1120-7.

48. Health DO. Report of the subgroup on bone health, working group on the nutritional status of the population of the Committee on Medical Aspects of Food and Nutrition Policy. In: Nutrition and bone health. London; 1998.

49. Wimalawansa SJ. Agriculture and chronic kidney disease. 2014. http://www.island.lk/index.php?page_cat=article-details\&page= article-details\&code_title $=98247, \mathrm{http}: / / \mathrm{www}$.dailynews.lk/fea tures/knocking-kidney-killer, http://www.sundayobserver.lk/ 2013/11/24/fea06.asp. Accessed 4 June 2014.

50. Bandara JM, Wijewardena HV, Seneviratne HM. Remediation of cadmium contaminated irrigation and drinking water: a large scale approach. Toxicol Lett. 2010;198(1):89-92.

51. Heiri C, et al. Forty years of natural dynamics in Swiss beech forests: structure, composition, and the influence of former management. Ecol Appl. 2009;19(7):1920-34.

52. Dissanayake CB, Chandrajith R. Medical geology in tropical countries with special reference to Sri Lanka. Environ Geochem Health. 2007;29(2):155-62.

53. Tennakone K, Wickramanayake S. Aluminium leaching from cooking utensils. Nature. 1987;325(6101):202. 
54. Partanen S. Inhibition of human renal acid phosphatases by nephrotoxic micromolar concentrations of fluoride. Exp Toxicol Pathol. 2002;54(3):231-7.

55. Ileperuma OA, Dharmagunawardhane HA, Herath KPRP. Dissolution of aluminium from sub-standard utensils under high fluoride stress: a possible risk factor for chronic renal failure in the North-Central Province. J Natl Sci Found Sri Lanka. 2009;37(3):219-22.

56. Schwartz A, Truman C. Chemistry in context. The wonder of water. Dubuque: W.M.C Brown; 1997.

57. DOW. Division of surface water-Ohio Lake Erie phosphorus task force final report. Ohio Environmental Protection Agency; 2010 p. 109.

58. Holt MS. Sources of chemical contaminants and routes into the freshwater environment. Food Chem Toxicol. 2000;38(1 Suppl):S21-7.

59. Noyes PD, et al. The toxicology of climate change: environmental contaminants in a warming world. Environ Int. 2009;35(6):971-86.

60. Desalegn B, et al. Mycotoxin detection in urine samples from patients with chronic kidney disease of uncertain etiology in Sri Lanka. Bull Environ Contam Toxicol. 2011;87(1):6-10.

61. Paerl HW, Otten TG. Harmful cyanobacterial blooms: causes, consequences, and controls. Microb Ecol. 2013;65(4):995-1010.

62. Langston CE, Heuter KJ. Leptospirosis. A re-emerging zoonotic disease. Vet Clin N Am Small Anim Pract. 2003;33(4):791-807.

63. Bezirtzoglou C, Dekas K, Charvalos E. Climate changes, environment and infection: facts, scenarios and growing awareness from the public health community within Europe. Anaerobe. 2011;17(6):337-40.

64. McBride AJ, et al. Leptospirosis. Curr Opin Infect Dis. 2005;18(5):376-86.

65. Smith EA, Oehme FW. The biological activity of glyphosate to plants and animals: a literature review. Vet Hum Toxicol. 1992;34(6):531-43.

66. Multiple-Authors. Glyphosate does not cause CKDu. 2014 http://www.island.lk/index.php?page_cat=article-details\&page= article-details\&code_title=100347, http://www.island.lk/index. php?page_cat $=$ article-details $\&$ page $=$ article-details $\&$ code_title $=$ 98864, http://www.island.lk/index.php?page_cat=article-details \&page=article-details\&code_title $=101248, \quad$ http://www.island. $1 \mathrm{k} /$ index.php?page_cat $=$ article-details $\&$ page $=$ article-details $\&$ code_title=100405, http://www.island.lk/index.php?page_cat= article-details\&page $=$ article-details\&code_title $=99203, \quad$ http:// www.island.lk/index.php?page_cat=article-details\&page=articledetails\&code_title=101392, http://www.lankaweb.com/news/ items/2014/07/06/there-is-no-scientific-evidence-that-herbicideglyphosate-causing-chronic-kidney-disease-of-multi-factorial-ori gin-ckd-mfo-in-sri-lanka/. Accessed 4 June 2014.

67. Zejda JE, McDuffie HH, Dosman JA. Epidemiology of health and safety risks in agriculture and related industries. Practical applications for rural physicians. West J Med. 1993; 158(1):56-63.

68. van der Hoek W, Konradsen F. Risk factors for acute pesticide poisoning in Sri Lanka. Trop Med Int Health. 2005;10(6): 589-96.

69. Eddleston $\mathrm{M}$, et al. Severe propanil [N-(3,4-dichlorophenyl)propanamide] pesticide self-poisoning. J Toxicol Clin Toxicol. 2002;40(7):847-54.

70. Pages W. Propanil and Agrochemicals. 1993. http://pmep.cce. cornell.edu/profiles/extoxnet/metiram-propoxur/propanil-ext. html, http://curriculum.toxicology.wikispaces.net/2.2.7.3.2+Pro panil?f=print). Accessed 4 June 2014.

71. Stockholm-convention, the convention is intended to protect human health and the environment from persistent organic pollutants (POPs). 2001. http://chm.pops.int/Portals/0/docs/pub lications/sc_factsheet_001.pdf. Accessed 4 June 2014.

72. European-Union-Council, Regulation (EC) No: 850/2004 of the European Parliament and the Council. 2004. http://eur-lex. europa.eu/LexUriServ/LexUriServ.do?uri=OJ:L:2004:158: 0007:0049:EN:PDF. Accessed 4 June 2014.

73. Buser HR, et al. Rapid anaerobic degradation of toxaphene in sewage sludge. Chemosphere. 2000;40(9-11):1213-20.

74. Chaidou CI, et al. Formation of chloroform by aqueous chlorination of organic compounds. Chemosphere. 1999;39(4): 587-94.

75. Hoekstra EJ, de Leer EWB, Brinkman UAT. Chloroform concentration gradients in soil air and atmospheric air, and emission fluxes from soil. Atmos Environ. 2001;35:61-70.

76. Cheng WH, Hsu SK, Chou MS. Volatile organic compound emissions from wastewater treatment plants in Taiwan: legal regulations and costs of control. J Environ Manag. 2008;88(4): 1485-94.

77. Lionte C. Lethal complications after poisoning with chloroform-case report and literature review. Hum Exp Toxicol. 2010;29(7):615-22.

78. Yaqoob M, Bell GM. Occupational factors and renal disease. Ren Fail. 1994;16(4):425-34.

79. Toxicological profile for chloroform. U.S. Department of Health and Human Services, Public Health Service Agency for Toxic Substances and Disease Registry. 1997.

80. Fang C, Behr M, Xie F, Lu S, Doret M, Luo H, Yang W, Aldous $\mathrm{K}$, Ding $\mathrm{X}, \mathrm{Gu} \mathrm{J}$. Mechanism of chloroform-induced renal toxicity: noninvolvement of hepatic cytochrome P450-dependent metabolism. Toxicol Appl Pharmacol. 2008;227(1):48-55.

81. Sun YX, et al. Persistent organic pollutants in marine fish from Yongxing Island, South China Sea: levels, composition profiles and human dietary exposure assessment. Chemosphere. 2014;98:84-90.

82. Speck-Planche A, et al. Predicting multiple ecotoxicological profiles in agrochemical fungicides: a multi-species chemoinformatic approach. Ecotoxicol Environ Saf. 2012;80:308-13.

83. Pereira Pinto LM, Boysielal K, Siung-Chang A. Pesticide regulation, utilization, and retailers' selling practices in Trinidad and Tobago, West Indies: current situation and needed changes. Rev Panam Salud Publica. 2007;22(2):83-90.

84. Bolognesi C. Genotoxicity of pesticides: a review of human biomonitoring studies. Mutat Res. 2003;543(3):251-72.

85. Zaim M, Guillet P. Alternative insecticides: an urgent need. Trends Parasitol. 2002;18(4):161-3.

86. Orantes CM, et al. Chronic kidney disease and associated risk factors in the Bajo Lempa region of El Salvador: nefrolempa study, 2009. MEDICC Rev. 2011;13(4):14-22.

87. Zhang XJ, Lai TB, Kong RY. Biology of fluoro-organic compounds. Top Curr Chem. 2012;308:365-404.

88. Sarkar A, et al. Sustainability of current agriculture practices, community perception, and implications for ecosystem health: an Indian study. EcoHealth. 2011;8(4):418-31.

89. Ames BN, Gold LS. Natural chemicals, synthetic chemicals, risk assessment, and cancer. Princess Takamatsu Symp. 1990;21:303-14.

90. Sharma CB, Sahu RK. Cytogenetic hazards from agricultural chemicals. I. A preliminary study on the responses of root meristems to exotoxin from Bacillus thuringiensis a constituent of a microbial insecticide, thuricide. Mutat Res. 1977;46(1):19-26.

91. Vinetz JM. Leptospirosis. Curr Opin Infect Dis. 2001;14(5):527-38.

92. Jiang $\mathrm{Y}$, et al. Immunoregulatory and anti-HIV-1 enzyme activities of antioxidant components from lotus (Nelumbo nucifera Gaertn) rhizome. Biosci Rep. 2011;31(5):381-90. 
93. Sim FG. Pesticides in Sri Lanka: documentation of selected literature and legal aspects, vol. 76. Colombo: Friedrich-EbertStiftun; 1989.

94. Taylor GJ. Pesticide use in Sri Lanka, in information from a study visit, March/April 1999. UK: Environment Group, Institute of Aquaculture: University of Sterling, UK; 1999.

95. Raksanam B, et al. Multi-approach model for improving agrochemical safety among rice farmers in Pathumthani, Thailand. Risk Manag Healthc Policy. 2012;5:75-82.

96. Naito R, et al. Effects of water management, connectivity, and surrounding land use on habitat use by frogs in rice paddies in Japan. Zool Sci. 2012;29(9):577-84.

97. Babiker IS, et al. Assessment of groundwater contamination by nitrate leaching from intensive vegetable cultivation using geographical information system. Environ Int. 2004;29(8): 1009-17.

98. Magkos F, Arvaniti F, Zampelas A. Organic food: buying more safety or just peace of mind? A critical review of the literature. Crit Rev Food Sci Nutr. 2006;46(1):23-56.

99. Castleden WM, et al. The mining and burning of coal: effects on health and the environment. Med J Aust. 2011;195(6):333-5.

100. Mertens TE, et al. Determinants of water quality, availability and use in Kurunegala, Sri Lanka. Trop Med Parasitol. 1990;41(1):89-97.

101. Programme APP. Symposium on population distribution. Asian Pac Popul Progr News. 1980;9(1-2):18.

102. Gunawardena RS. Demographic and socio-economic situation in a new settlement in Sri Lanka: a study of system C of the Mahaweli Development Programme. Sir Lanka J Popul Stud. 1998;1(1):65-84 (125).

103. Awumbila M, Momsen JH. Gender and the environment. Women's time use as a measure of environmental change. Glob Environ Change. 1995;5(4):337-46.

104. Amerasinghe FP, Indrajith NG. Postirrigation breeding patterns of surface water mosquitoes in the Mahaweli Project, Sri Lanka, and comparisons with preceding developmental phases. J Med Entomol. 1994;31(4):516-23.

105. Amerasinghe FP, Munasingha NB. A predevelopment mosquito survey in the Mahaweli Development Project area, Sri Lanka: immatures. J Med Entomol. 1988;25(4):286-94.

106. Lantz O, et al. Fluoride-induced chronic renal failure. Am J Kidney Dis. 1987;10(2):136-9.

107. Company R, et al. Using biochemical and isotope geochemistry to understand the environmental and public health implications of lead pollution in the lower Guadiana River, Iberia: a freshwater bivalve study. Sci Total Environ. 2008;405(1-3):109-19.

108. Holas J, Hrncir M. Integrated watershed approach in controlling point and non-point source pollution within Zelivka drinking water reservoir. Water Sci Technol. 2002;45(9):293-300.

109. Van der Hoek W, et al. Pesticide poisoning: a major health problem in Sri Lanka. Soc Sci Med. 1998;46(4-5):495-504.

110. Shipitalo MJ, et al. Impact of grassed waterways and compost filter socks on the quality of surface runoff from corn fields. J Environ Qual. 2010;39(3):1009-18.

111. WHO. Food safety issues associated with products from aquaculture. Report of a Joint FAO/NACA/WHO Study Group. World Health Organ Tech Rep Ser. 1999; 883: i-vii, 1-55.

112. Hossain MS, et al. Clinical solid waste management practices and its impact on human health and environment-a review. Waste Manag. 2011;31(4):754-66.
113. Haylamicheal ID, Dalvie MA. Disposal of obsolete pesticides, the case of Ethiopia. Environ Int. 2009;35(3):667-73.

114. Bridges TS, et al. Dredging processes and remedy effectiveness: relationship to the $4 \mathrm{Rs}$ of environmental dredging. Integr Environ Assess Manag. 2010;6(4):619-30.

115. Verta $\mathrm{M}$, et al. A decision framework for possible remediation of contaminated sediments in the River Kymijoki, Finland. Environ Sci Pollut Res Int. 2009;16(1):95-105.

116. Gomes Do Espirito Santo ME, et al. Investigation of deaths in an area of groundnut plantations in Casamance, South of Senegal after exposure to Carbofuran, Thiram and Benomyl. J Expo Anal Environ Epidemiol. 2002;12(5):381-8.

117. Rajasekaran LR, Blake TJ. New plant growth regulators protect photosynthesis and enhance growth under drought of jack pine seedlings. J Plant Growth Regul. 1999;18(4):175-81.

118. Neumann PM. Senescence of attached bean leaves accelerated by sprays of silicone oil antitranspirants. Plant Physiol. 1974;53(4):638-40.

119. Jeffree CE, Johnson RP, Jarvis PG. Epicuticular wax in the stomatal antechamber of sitka spruce and its effects on the diffusion of water vapour and carbon dioxide. Planta. 1971;98(1):1-10.

120. Salas P, et al. Growth retardation in children with kidney disease. Int J Endocrinol. 2013;2013:970946.

121. Gama-Axelsson T, et al. Serum albumin as predictor of nutritional status in patients with ESRD. Clin J Am Soc Nephrol. 2012;7(9):1446-53.

122. Wimalawansa SJ. Purification of contaminated water with reverse osmosis: effective solution of providing clean water for human needs in developing countries. J Emerg Technol Adv Eng. 2013;3(12):75-89.

123. Powe NR, Plantinga L, Saran R. Public health surveillance of CKD: principles, steps, and challenges. Am J Kidney Dis. 2009;53(3 Suppl 3):S37-45.

124. Hossain MP, et al. CKD and poverty: a growing global challenge. Am J Kidney Dis. 2009;53(1):166-74.

125. Shoham DA, et al. Kidney disease and the cumulative burden of life course socioeconomic conditions: the Atherosclerosis Risk in Communities (ARIC) study. Soc Sci Med. 2008;67(8): 1311-20.

126. Wimalawansa, SJ. Stigma of obesity: a major barrier to overcome. J Clin Transl Endocrinol 2014;1(3):73-76. doi:10.1016/j. jcte.2014.06.001.

127. Fujii H. CKD-MBD (chronic kidney disease-mineral and bone disorder). Effect of vitamin D on kidney and cardiovascular system. Clin Calcium. 2010;20(7):1045-50.

128. Wimalawansa SJ. Vitamin D everything you need to know. Homagama: Karunaratne \& Sons; 2012. ISBN 978-955-909894-2.

129. Hill BT. The establishment of criteria for "quiescence" in ageing human embryo cell cultures and their response to a proliferative stimulus. Gerontology. 1977;23(4):245-55.

130. Venuthurupalli SK, et al. CKD.QLD: chronic kidney disease surveillance and research in Queensland, Australia. Nephrol Dial Transplant. 2012;27(3):45-139.

131. Wimalawansa SA, Rajapakse S, Wimalawansa SJ. Environmental Pollution Related Escalating Occupational Diseases. Int Res J Pub Environ Health Res (submitted). 